\title{
Réflexions d'un métallurgiste sur le lien entre les ressources et le développement régional
}

Martin Taylor, Msc.

Né en Angleterre, j’y ai fait mes études en métallurgie. Dans les années ' 60 , je suis parti vivre en Ontario, Canada, pour ensuite me rendre au Québec en 1972.

Ma carrière s'étend sur une période de 48 ans, toujours associée à l'industrie des métaux. J'ai

J'entends comme «ressources» les aspects suivants: les matières premières; la sylviculture; l'agriculture; l'énergie (eau-

\section{Ma carrière dans une fonderie d'acier}

Sheffield, ville réputée pendant des siècles pour son industrie de la coutellerie, est devenue prospère grâce à sa richesse en eau, qui s'y trouve en abondance. Cinq rivières coulent à travers la ville, et de nombreux barrages permettent d'alimenter les forges. Le charbon et le minerai de fer étaient extraits des mines locales. Au $19^{\text {ième }}$ siècle, lors de la révolution industrielle, les nouvelles méthodes de production comme le procédé de Bessemer, développé à Sheffield, ont permis la croissance des aciéries. Par contre, la qualité et la disponibilité du minerai de fer nécessitaient son importation.

Avant la fin de la Seconde Guerre mondiale, l'industrie s'est diversifiée, se lançant dans la production de produits de toutes sortes : outils, pièces forgées, pièces coulées, barres, plaques et ainsi de suite. Évidemment, la coutellerie restait encore un produit privilégié. Le pays avait désespérément besoin d'être reconstruit, débuté à Sheffield, Angleterre, dans une fonderie produisant des aciers de haute gamme, pour ensuite fermer la boucle au Saguenay, Québec, dans l'industrie de l'aluminium. Le fil en commun pendant ces années ? Je crois que c'est la disponibilité et l'exploitabilité des ressources. électricité); le pétrole et le charbon; et, dernier aspect très important, les ressources humaines.

mais les matériaux bruts se faisaient rares. Par contre, contrairement à l'époque de la Grande Dépression qui avait eu lieu vingt ou trente ans plus tôt, il y avait suffisamment de travail pour la population locale.

Malgré la forte demande en produits pendant et immédiatement après la Deuxième Guerre mondiale, l'industrie n'a pas investi dans les procédés plus modernes, tel que LinzDonawitz, développé en Autriche. Il n'y a pas eu de développement ni de nouveaux marchés et ce, malgré la bonne quantité et la qualité du charbon, une ressource nécessaire utilisée comme réducteur dans les procédés d'acier et comme carburant pour l'électricité. Le résultat de ces erreurs ? Madame Thatcher a provoqué la fermeture de toute l'industrie de l'acier à Sheffield dans les années '80. La compétition mondiale de plusieurs pays devenait une réalité et de nouvelles ressources étaient trouvées et développées. 


\section{Ma carrière en production d'alliages de manganèse et de silicium}

En 1965, ma carrière dans une vieille industrie qui n'investissait pas suffisamment pour rester compétitive n'était pas prometteuse. J'ai donc décidé d'émigrer au Canada pour travailler comme ingénieur en développement au sein d'un groupe en recherche et développement (R\&D) dans le secteur «Métaux et carbone », pour la compagnie Union Carbide Canada Ltd, en Ontario. Un de mes mandats était d'étudier la stratégie de production des alliages de chrome, du silicium, du manganèse, du vanadium et autres métaux pour la compagnie. Cette étude a démontré entre autres que pour une compagnie qui n'est pas le propriétaire majoritaire des ressources de minerais et d'énergie, le retour sur l'investissement n'est pas toujours assuré.

Nous avons étudié différentes options dans toute l'Amérique du Nord. Par contre, le gouvernement de l'Ontario a pris la décision de ne plus fournir d'électricité à bas prix aux industries de haute consommation. Il faut se rappeler que les usines près de Niagara Falls profitaient de bons tarifs depuis le début du $20^{\text {ième }}$ siècle. Le résultat a été qu'il fut décidé de relocaliser la production des alliages de Welland. Il y avait de l'électricité en abondance MAIS SEULEMENT À CE MOMENT-LÀ ! Et pour combien de temps ?

L'usine de Beauharnois, Québec, a été choisie pour la production des alliages de manganèse ; on m'a donc demandé de déménager au
Québec et de superviser la construction d'une nouvelle usine. Hydro-Québec a confié un contrat d'électricité d'une durée de 15 ans et une garantie de bas prix pour les 10 premières années. De plus, les gouvernements fédéral et provincial ont subventionné le projet de 15 millions de dollars, à un taux de $10 \%$ du coût total.

\section{Les usines les plus performantes peuvent produire de l'aluminium pour environ $12500 \mathrm{kWh} /$ tonne}

Il est à noter que la production d'aluminium liquide requiert plus d'énergie que la production de silicium. Les usines les plus performantes peuvent produire de l'aluminium pour environ $12500 \mathrm{kWh} /$ tonne, ce qui contraste avec les électrolyses qui, dans les années '40, produisaient de l'aluminium avec une consommation d'environ $20000 \mathrm{kWh}$ /tonne. Un nouveau procédé, le AP50 de Rio Tinto Alcan (RTA), devrait bientôt être implanté pour améliorer l'efficacité et la capacité comparativement au AP 35 de Pechiney. Aussi faut-il noter que la combinaison d'énergie, de minerais et des réducteurs (dans le cas des fours à arc électrique) constituent les ressources clés pour la production des métaux. Il suffit de mentionner la production d'aluminium et de manganèse ; ces ressources représentent environ $60 \%$ des coûts.

\section{Ma carrière comme dirigeant dans l'industrie des métaux}

Avec l'installation d'une nouvelle usine à Beauharnois en 1972, je pouvais voir de près à quel point l'industrie des métaux avait changé. L'usine comptait le plus grand four au monde, fourni par les Japonais. Ces derniers s'étaient lancés dans la conception de fours, un domaine auparavant occupé par les Américains et les Européens. Plus tard, j'ai accepté un poste de 
directeur en ingénierie de l'usine produisant des alliages de silicium et de manganèse. Vers la fin des années ' 70 , non seulement le prix de l'énergie avait-il grimpé mais aussi le militantisme des syndicalistes, qui a conduit à la fermeture de l'usine de silicium de Beauharnois (les premiers fours ont été construits en 1936).

De plus, à cette époque, Alcan changeait sa stratégie au niveau de l'achat de silicium métallique. Dorénavant, les fournisseurs se trouvaient outre-mer. Union Carbide, le fournisseur canadien de longue date d'Alcan et voisin géographique, venait de perdre son meilleur client.

Vers la fin des années '70, Union Carbide avait fait une étude pour évaluer sa stratégie dans un monde changeant et avait pris la décision, en 1984, de vendre tous les actifs qu'elle possédait dans le secteur des métaux à travers le monde. Une nouvelle compagnie a été créée, Elkem Métal Canada, et j'en devins le président.

Elkem a décidé de transférer à Chicoutimi, dans la région du Saguenay au Québec, toute la production des alliages de silicium. À ce moment, la production de silicium métallique (utilisé comme alliage avec l'aluminium) était déjà arrêtée. La compagnie était propriétaire de Smelter Power, une mini-centrale qui, localisée dans la «Veille Pulperie» de Chicoutimi, répondait à une bonne partie de ses besoins en énergie à un très bas coût.

L'usine fonctionne toujours aujourd'hui. Sans cette ressource, et dû aux coûts élevés générés par l'importation du charbon et le transport du produit finit vers les aciéries de l'Ontario et des États-Unis, l'usine ne pourrait pas être rentable (opinion de l'auteur).
En 1991, Elkem, malgré la rentabilité de l'usine de manganèse à l'époque, a malheureusement mis fin à ses activités à Beauharnois. La réalité de la compétition mondiale obligeait la compagnie à revoir sa stratégie. La production a été maintenue aux États-Unis, plus précisément dans la ville de Marietta, où le gouvernement américain donnait à très bons prix les minerais de manganèse de ses stocks stratégiques. La production s'est aussi poursuivie en Norvège, où les lois sur la mise à pied sont plus strictes qu'au Québec. Elkem est un exemple typique de propriétaire étranger ayant adopté comme stratégie d'abandonner ses investissements pour maintenir à n'importe quel prix les emplois dans son pays. Un autre exemple récent est l'usine de Norsk Hydro à Bécancour, qui a fermé ses portes en 2007. À Beauharnois, la production de métaux n'a pas survécu à 60 années. À Sheffield, c'est de siècles de production dont il s'agit! Depuis un certain temps, la tendance démontre que les cycles de vie sont de plus en plus courts et que le monde change vite !
Elkem est un exemple typique de propriétaire étranger ayant adopté comme stratégie d'abandonner ses investissements pour maintenir à n'importe quel prix les emplois dans son pays
Pour conclure cette partie de mon histoire, il faut souligner qu'en 1988, Elkem avait aussi étudié les perspectives d'avenir pour les producteurs de métaux dans leur champ d'activité. La conclusion était évidente. Le mouvement de transfert vers les pays en voie de développement se poursuivra, une tendance qui s'est confirmée au cours des deux dernières décennies. Toutes les nouvelles productions sont concentrées au MoyenOrient, en Afrique et en Asie. 


\section{Ma carrière dans l'industrie de l'aluminium}

Après 33 ans dans la production de métaux et d'alliages, j'ai accepté en 1992 un poste de vice-président en marketing international au sein d'une petite entreprise au Saguenay, STAS, pour l'aider à s'ouvrir sur le marché international. À l'époque, j'étais connu du personnel de direction d'Alcan, qui était une compagnie voisine d'Elkem à Chicoutimi et à Beauharnois ainsi qu'un client d'Elkem.

Le fondateur de STAS, Pierre Bouchard, a été bien inspiré lorsqu'il réalisa, lors d'une récession en 1982, que de nouveaux emplois régionaux devaient être créés dans des champs d'activités autres que les industries traditionnelles. Pourquoi importer ces équipements sophistiqués d'autres pays quand on peut les fabriquer soi-même?

En 1989, la compagnie avait commencé à fabriquer sous licence des équipements d'Alcan pour le traitement de l'aluminium dans les alumineries et les fonderies. Grâce à une solide performance du groupe $\mathrm{R} \& \mathrm{D}$ d'Alcan, STAS pouvait créer de nouveaux emplois dans la région. Le gouvernement du Québec a décidé d'accorder à STAS une subvention de deux ans pour que cette petite compagnie puisse s'ouvrir sur le marché international. Il faut retenir le fait que les efforts en marketing exigent du temps, souvent de trois à cinq ans, avant que des commandes ne puissent être reçues.

Depuis les 15 dernières années, la compagnie a triplé ses ventes et emploie plus de 135 personnes. STAS est reconnue mondialement comme fournisseur d'équipements spécialisés utilisés pour améliorer la qualité et l'efficacité des procédés du traitement de métal liquide.

J'ai beaucoup voyagé pour visiter les clients et participer à des conférences, et j'ai énor- mément appris pendant ces années. Ce travail en marketing, mené en parallèle avec des contrats en consultation pour l'Argentine, par exemple, ou encore Europe de l'Est, m'a permis de tirer certaines conclusions. Je rajoute que je ne suis pas la seule personne qui soit arrivée à ces conclusions, mais je crois qu'il faut les répéter.

En 1992, Alcan est arrivée à la même conclusion : pour faire un profit acceptable, il faut être propriétaire de la bauxite et de sa conversion en alumine. Une fois cette ressource sous son contrôle, il est possible de convertir l'alumine en aluminium en utilisant une énergie (électricité) à bon prix. Ici encore, il est préférable d'être propriétaire de cette ressource énergétique (comme au Québec, par exemple). Les deuxième et troisième conversions en produits finis ne peuvent être rentables que si le métal primaire est disponible à bon prix. Le profit se fait principalement dans le secteur primaire; la stratégie adoptée par Alcan dans les années '90 a donc été de vendre les installations de transformation qu'elle possédait un peu partout au monde.

\section{Il ne fait aucun doute que Rio Tinto Alcan (RTA) ne peut pas opérer ses usines au Saguenay s'il n'y a plus d'électricité bon marché}

Il ne fait aucun doute que Rio Tinto Alcan (RTA) ne peut pas opérer ses usines au Saguenay s'il n'y a plus d'électricité bon marché (voir mes commentaires sur Elkem Métal, un peu plus haut), et la même chose est vraie pour les autres producteurs au Québec, comme Alcoa. Toutes les nouvelles usines construites depuis les années '70 ont pu l'être grâce aux tarifs avantageux accordés par Hydro-Québec, mais aujourd'hui, les choses ont changé, et le prix de l'électricité disponible 
est moins compétitif que les prix en vigueur dans d'autres régions du globe.

Récemment, j'ai fait une étude pour une nouvelle compagnie afin de déterminer la possibilité d'un projet pour la production des alliages de manganèse. Le requérant avait constaté que le prix des métaux était à son plus haut depuis plusieurs années et que la demande de la Chine était en croissance. Malgré cette perception, il a été démontré que le coût de l'électricité était encore trop élevé à tarif « $\mathrm{L}$ ». De plus, il faut importer le minerai à des coûts trop élevés.

\section{$\underline{\text { Le portrait de l'énergie (électricité) }}$}

1. Aujourd'hui, il est un fait reconnu que la planète ne peut plus dépendre de l'énergie à bas prix, la majorité étant fournie par les carburants fossiles. De plus, ces ressources non renouvelables causent des changements climatiques. Les trois industries dans lesquelles j'ai travaillé, c'est-à-dire une fonderie au RoyaumeUni, l'industrie des ferro-alliages et celle de l'aluminium au Canada, dépendaient toutes de l'énergie à bas prix pour leur développement, mais les prix ont augmenté et ont conduit à la fermeture des usines. La production est maintenant concentrée dans des pays où le prix de l'énergie est maintenant le moins élevé. Cela ne peut continuer ainsi !

2. Aujourd'hui, la Chine produit environ 10000000 tonnes par année, ce qui compte pour presque $30 \%$ de la production mondiale en aluminium comparativement à 2 millions de tonnes il y a 10 ans. Faute d'énergie à bas prix, le gouvernement est en train de freiner la croissance de cette industrie.

3. Aussi, le Moyen-Orient compte parmi les régions où la croissance de l'industrie de l'aluminium est très forte grâce à la
La consommation et la production d'aluminium augmentent à un rythme constant, autour de 3 à $4 \%$ par année. Soulignons cependant qu'avec l'avance de la modernisation et de l'automatisation, le nombre d'employés au sein de l'entreprise RTA au SaguenayLac-St-Jean continue de diminuer. Je n'ai pas de données précises à présenter, mais en me basant sur des conversations que j'ai eues avec le personnel de RTA, les emplois étaient au nombre d'environ 10000 il y a 20 ans comparativement à moins de 6000 emplois aujourd'hui.

disponibilité du gaz naturel à bas prix. Pourtant, il y a lieu d'être préoccupés. Un élément qui participe largement à la croissance rapide de l'industrie se trouve dans la région du Golf, au Moyen-Orient : de l'électricité à bas prix fournie principalement à partir de gaz naturel. Ces pays incluent les Émirats unis (Dubai et Abu Dhabi), Oman, Qatar et l'Arabie saoudite. Il importe aussi de noter que dans TOUS les cas, il y a appropriation par l'état, principalement par le biais de sociétés de portefeuille dans le pétrole et de compagnies d'investissement acquises par le gouvernement. Toutefois, la production de gaz en quantité illimitée (la source principale d'électricité pour les alumineries) commence à diminuer dans la région. Des rapports indiquent déjà qu'il faudra importer du charbon dans un avenir assez rapproché !

4. L'un des dilemmes auxquels les pays sont confrontés est le fait que la demande en électricité domestique soit toujours de plus en plus élevée dans plusieurs pays en développement. Par exemple, HydroQuébec subit des pressions pour exporter son électricité aux États-Unis, où les profits seront possiblement beaucoup plus 
élevés que si cette énergie était simplement vendue à une clientèle domestique. Un autre exemple est le Qatar, dans la région du Golfe, qui approvisionne l'Europe, le Japon, les États-Unis et bien d'autres pays en gaz naturel liquéfié, encore une fois avec des revenus beaucoup plus élevés que ce qu'il serait possible d'obtenir avec les alumineries locales. La Russie est de loin le plus important fournisseur de gaz pour l'Europe et se repose sur ses sources hydroélectriques pour approvisionner ses nouvelles fonderies.

5. À noter que dans presque tous les exemples indiqués plus haut, la fourniture en énergie se fait par une participation gouvernementale directe ou indirecte.

6. Plusieurs régions industrielles font montre d'une certaine exigence visant, comme c'est le cas de la région du Golfe, qui connaît une croissance économique rapide, à diversifier les ressources ou produits d'exportation autres que le pétrole ou le gaz naturel en préparation à un avenir où ces ressources non renouvelables se feront plus rares. À titre d'exemple, il suffit de mentionner Dubai, où le tourisme et le commerce sont en voie de remplacer les exportations en pétrole.

Je prédis que d'ici la prochaine décennie, les alumineries partout dans le monde n'auront plus accès à l'électricité à bon marché. Par contre, le recyclage se poursuivra en force. L'aluminium recyclé requiert moins de $10 \%$ en consommation d'énergie électrique comparativement à une fonderie qui produit une tonne d'aluminium. Comme exemple, citons la Chine, qui accroît considérablement ses opérations en aval par la transformation de l'aluminium pour la consommation domestique en feuilles métalliques, en lingots à canettes, en extrusions et ainsi de suite. Toutes les nouvelles usines du Moyen-Orient (incluant les expansions) recherchent activement des projets de transformation à long terme de l'aluminium en provenance des nouvelles fonderies. Bahrain en est un bon exemple, ayant atteint $60 \%$ de son objectif consistant à convertir l'aluminium primaire en produits secondaires dans les usines locales.

\section{La région du Saguenay et le Québec}

Dans mon esprit, il est clair que la région, tout autant que le Québec, n'a pas su encourager sérieusement l'industrie de la production d'aluminium de deuxième fusion. Selon un rapport émis par l'Association de l'aluminium en 2004, 23\% de l'aluminium brut est transformé en produits secondaires, ce qui représente un pourcentage beaucoup plus faible comparativement au Bahrain, par exemple. De plus, il y a eu une croissance dans la production de l'aluminium primaire depuis 2004, plus spécialement à Sept-Îles.
Mentionnons encore que la stratégie de RTA (Alcan à l'époque) poursuit toujours la même stratégie en vendant une énorme portion de ses opérations en aval par la création de Novelis (maintenant propriété d'Hindalco, en Inde), après avoir acheté Alusuisse et Pechiney. Ceci démontre que ces décisions stratégiques ont été prises selon l'argument que j'ai introduit plus tôt et voulant que la marge de profit principale se trouve dans la conversion de minerai en métal, NON dans sa transformation en produits en aval. 
J'ai aussi mentionné plus tôt que pour la production de métaux, il faut posséder le minerai et avoir la capacité de le convertir à bas prix (détention de la production d'énergie) en métal primaire. C'est sûrement la raison pour laquelle Alcan (RTA), récemment acquise par Rio Tinto d'Australie/R.-U., ne se laissera pas persuader de changer sa stratégie SANS QUE LE GOUVERNEMENT NE DÉCIDE D'INTERVENIR. Dans la région du Saguenay, l'intervention gouvernementale a été d'assurer de l'énergie électrique à long terme et à bon marché afin de convaincre Alcan d'investir dans une version améliorée de la fonderie conçue par Pechiney, le AP50.

\section{Fermetures d'usines}

J'ai travaillé dans trois grandes usines de production de métaux au Royaume-Uni et au Canada. Les trois usines ont dû cesser leur production dû aux coûts de production élevés causés par des coûts en énergie, en maind'oeuvre et en matières premières soit trop

\section{Manufacturiers d'équipements}

STAS est l'exemple d'une compagnie qui a réussi à exploiter les opportunités du marché mondial en tant que compagnie spécialisée dans la conception et l'exportation d'équipements à la fine pointe de la

\section{$\underline{\text { Production d'aluminium primaire }}$}

En ce qui touche la production d'aluminium, il est plus profitable de produire de l'aluminium à partir de minerais que de transformer le produit brut pour le marché de consommation. Au cours des cinq dernières années, STAS, avec environ $70 \%$ de ses ventes en exportation, a constaté combien il est difficile pour grand nombre de ses clients de survivre simplement dans la transformation de
Un autre exemple récent d'intervention gouvernementale est la production de parechocs d'automobiles au Saguenay, par l'usine RTA. Au moment d'écrire cet article, une annonce venait juste d'être émise officiellement pour annoncer que l'usine serait fermée, ce qui n'est pas réellement surprenant étant donné que l'aluminium liquide produit dans la région du Saguenay n'a jamais été fourni à cette usine. Les conditions du marché ainsi que les coûts de transport ont été cités comme raisons principales de cette décision.

élevés ou encore dépassés comparativement aux coûts encourus par les fournisseurs outremer. En d'autres mots, les usines étaient incapables de tirer leur profit de l'exploitation des ressources.

technologie. Le Saguenay compte aussi quelques autres compagnies qui exportent leurs produits avec succès tout en créant de l'emploi dans le secteur des services et de la fabrication spécialisée.

l'aluminium en produits finis. En fait, plusieurs usines ont dû fermer leurs portes ou luttent pour leur survie, particulièrement en Europe et en Amérique du Nord. La majorité des ventes de STAS se concluent principalement avec les alumineries, spécialement les nouvelles, plutôt que dans les secteurs secondaires. 


\section{Intervention gouvernementale}

Un peu partout dans le monde, les projets visant l'exploitation des ressources ont pu voir le jour grâce à l'intervention gouvernementale. Il est fort probable que cette tendance se maintienne pendant quelque temps encore, du moins pendant que les coûts en investissement seront élevés. Par contre, il arrive dans certains cas que l'intervention gouvernementale ne soit pas la solution pour les investisseurs.

\section{$\underline{\text { L'avenir }}$}

Le développement du Saguenay a été possible grâce à l'exploitation de son eau (électricité) et de ses forêts. Par contre, très peu d'efforts ont été investis au cours du dernier siècle pour ajouter de la valeur aux produits vendus hors de la région, ce qui a eu pour effet de lui faire perdre la course contre la compétition mondiale, qui a su fournir à des coûts beaucoup plus avantageux des produits provenant des deux mêmes secteurs.

Pour vérifier cette réalité, je propose deux étapes, basées sur les points suivants :

1. RTA devrait confirmer qu'il leur est impossible, avec la structure de coûts actuelle, de faire un profit raisonnable en investissant dans les opérations en aval. Que faudrait-il pour que cela puisse se produire? Une implication gouvernementale, ou des amortissements cumulés spéciaux sur les dépenses en capital, ou peut-être des concessions par la maind'oeuvre? Il ne fait aucun doute que le point essentiel est que l'aluminium liquide soit fourni au coûtant (ET NON AU PRIX DU LME). Comment persuader RTA de participer à un tel exercice est une question difficile, mais l'exercice doit quand même se faire.

2. RTA devrait confirmer qu'il n'est pas dans ses intérêts stratégiques de
Quel prix a dû payer le gouvernement provincial pour permettre à RTA d'exploiter les ressources énergétiques (eau et électricité) pendant les 50 prochaines années sans aucune chance de prendre en compte d'autres types d'utilisation de cette électricité ?

Je crois que la région du Saguenay devrait, par la voix et l'action de ses politiciens et de la population, faire un exercice très simple pour vérifier et valider le fait qu'il est irréaliste de continuer à se fier à RTA comme source d'emploi dans les opérations en aval. La région doit diversifier ses champs d'activité.

développer une industrie secondaire importante. Ses principaux marchés se retrouvent outre-mer, plus près du marché de consommation de l'Europe, de l'Asie et des États-Unis.

3. Si RTA ne peut justifier la transformation d'un large pourcentage de l'aluminium de ses fonderies en produits secondaires, il est alors peu probable que d'autres compagnies puissent entrer en jeu, certainement pas des compétiteurs, pour s'emparer de l'aluminium liquide et le transformer!

4. S'il est démontré que RTA et/ou d'autres ne peuvent pas développer une industrie

RTA devrait confirmer qu'il n'est pas dans ses intérêts stratégiques de développer une industrie secondaire importante 
secondaire suffisamment importante, la région devra alors tourner son attention vers d'autres types de développement afin de développer des produits à partir de ses ressources locales comme la forêt, par exemple, qui pourrait permettre la production de nouveaux médicaments pour les compagnies pharmaceutiques.

À mon avis, le message est clair pour la région. Nous ne pouvons pas dépendre des industries primaires pour la création de nouveaux emplois dans les années à venir. Nous devons plutôt nous tourner vers les secteurs de services et de fabrication spécialisée pour leur développement à long

\section{Une étape radicale consisterait à} nationaliser les stations de production d'électricité de la région
5. Une étape radicale consisterait à nationaliser les stations de production d'électricité de la région, de payer une compensation et de vendre l'énergie en excès aux Américains! Par contre, les revenus générés seraient-ils réinvestis dans la région du Saguenay? J'en doute.

terme et former nos ressources humaines (la main-d'oeuvre) pour nous adapter à la nouvelle réalité du marché international. La matière grise, c'est-à-dire le savoir et les connaissances, est maintenant un requis dans la vaste majorité des nouveaux emplois. Il suffit de penser aux secteurs hautement rémunérés comme l'ingénierie, la recherche et le développement ainsi que les emplois dans le domaine médical, qui ne sont que quelques exemples de succès. 


\section{Formulaire d'abonnement}

